\title{
Avaliação da Inteligência de Crianças Deficientes Visuais: Proposta de Instrumento
}

Evaluating Intelligence of Children with Visual

Disabilities: Instrument Proposal

Evaluación de la Inteligencia de Niños Deficientes Visuales: Propuesta de Instrumento

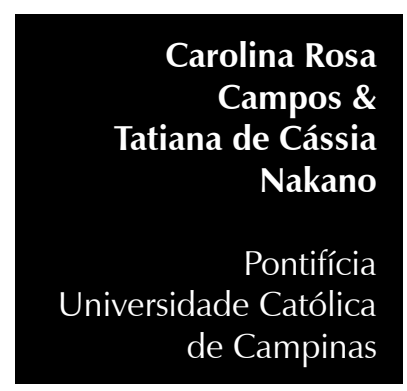

http://dx.doi.org/10.1590/1982-3703000272013
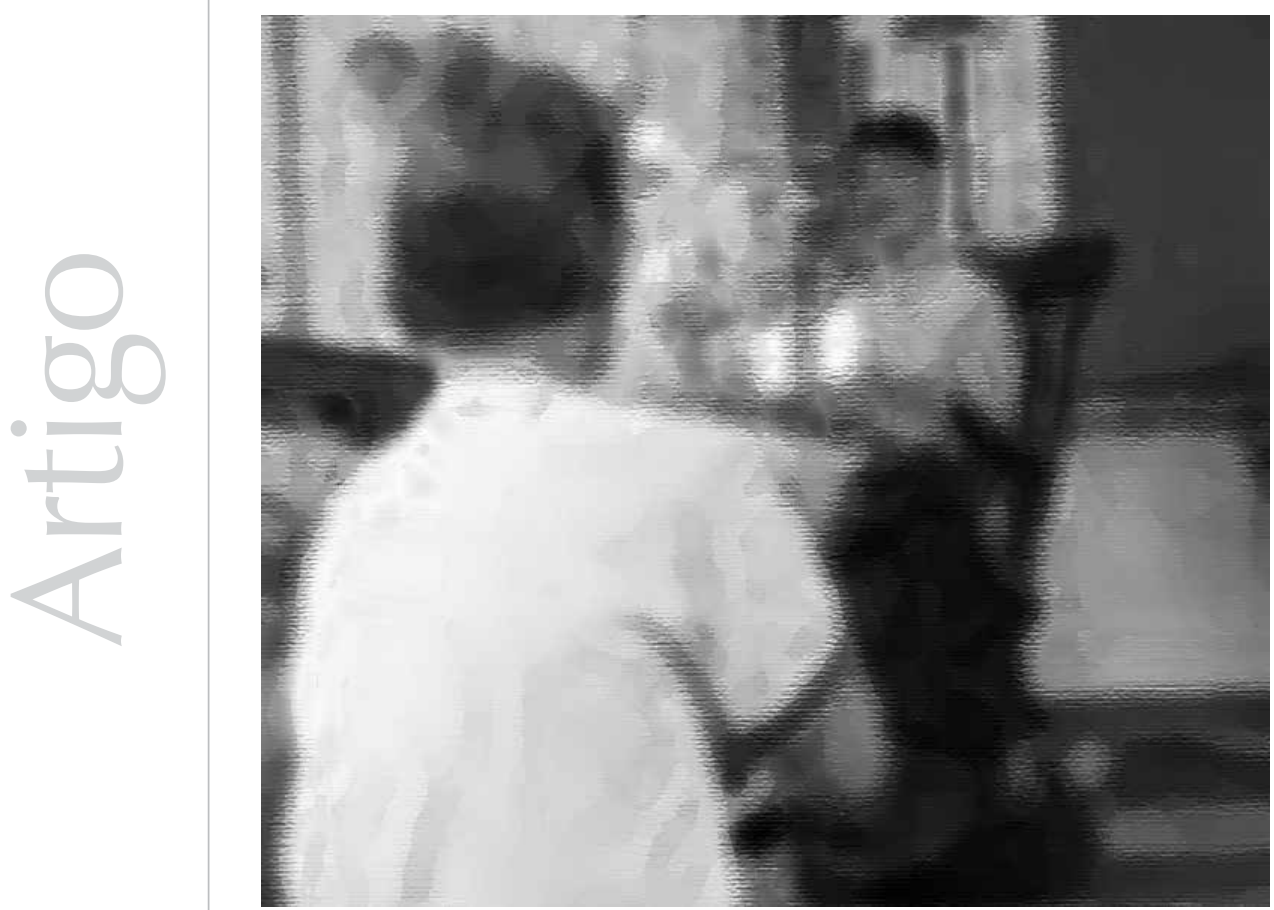
Resumo: Diante da lacuna existente na avaliação cognitiva de populações especiais, esta pesquisa teve como objetivo construir três subtestes (Verbal, Memória e Lógico-espacial) para avaliação da inteligência de crianças deficientes visuais, baseados no modelo de Cattel-Horn-Carroll (CHC). Estudo piloto foi desenvolvido visando verificar a adequação dos subtestes em 14 crianças deficientes visuais de 7 a 12 anos $(M=10,28$ anos; $\mathrm{DP}=1,58$ ), sendo seis meninas e oito meninos, dez com baixa visão, oito com deficiência congênita e duas com adquirida, e quatro com cegueira, duas com adquirida e duas com congênita. Avaliamos as medidas de acertos e tempo de execução no Verbal, acertos, quantidade de pares abertos e tempo para o Memória e acertos e tempo para o pensamento Lógico-espacial. Os resultados apontaram adequação dos subtestes à população, notando-se melhor desempenho das crianças com deficiência congênita e melhor desempenho das crianças com baixa visão. Estudos com amostras maiores poderão investigar as propriedades psicométricas dos subtestes.

Palavras-chave: Inteligência. Medidas de inteligência. Deficiente visual. Cognição.

Abstract: Given the gap in cognitive assessment of people with special needs, this research sought to build three subtests (Verbal, Memory and Logical) to assess the intelligence of visually impaired children, based on the Cattell-Horn-Carroll model (CHC). A pilot study was developed to verify the adequacy of the subtests in 14 visually impaired children ages 7-12 ( $M=10.28$ years, $S D=1.58)$, six girls and eight boys, ten had low vision (eight with cognitive disabilities and two with acquired), and four with blindness (two with congenital and two acquired), measuring right answers and time taken Verbal; right answers, number of open pairs and time in Memory; and right answers and time taken in Logic. The results indicated the suitability of the subtests to this population, noting best performance in children with congenital disabilities and better performance of children with low vision. Larger studies could investigate the psychometric properties of the subtests.

Keywords: Intelligence. Measuring intelligence. Visually disabled. Cognition.

Resumen: Ante el hueco existente en la evaluación cognitiva de poblaciones especiales, esta investigación ha tenido como objetivo construir tres sub-testes (Verbal, Memoria y Lógico-espacial) para la evaluación de la inteligencia de niños deficientes visuales, basados en el modelo de Cattel-Horn-Carroll (CHC). El estudio piloto ha sido desarrollado objetivando verificar la adecuación de los sub-testes en 14 niños deficientes visuales entre 07 y 12 años $(M=10,28$ puntos; $D P=1,58)$, siendo seis niñas y ocho niños, diez con baja visión (ocho con deficiencia congénita y dos con adquirida) y cuatro con ceguera (dos con adquirida y dos con congénita) en las medidas de aciertos y tiempo de ejecución en el Verbal; aciertos, cantidad de pares abiertos y tiempo en el Memoria; y aciertos y tiempo en el Lógico-espacial. Los resultados han señalado una adecuación de los sub-testes a la población, notándose mejor desempeño de los niños con deficiencia congénita y mejor desempeño de los niños con baja visión. Estudios con muestras mayores podrán investigar las propiedades psicométricas de los sub-testes.

Palabras clave: Inteligencia. Medidas de Inteligencia. Discapacidad visual. Cognicion.

Historicamente, os testes de inteligência têm cumprido e continuarão a cumprir uma importante função na Psicologia, embora poucos instrumentos consigam representar a multiplicidade de aspectos que intervêm na cognição e no desempenho humano (Almeida, Guisande, Primi \& Ferreira, 2008). Dado esse desafio, a popularidade crescente nos últimos anos da teoria CHC (Cattell-Horn-Carroll) tem conduzido autores e editores de provas de inteligência à procura de adequação das suas baterias e escalas a esse modelo teórico, de modo a nortear os trabalhos de revisão das baterias de inteligência disponíveis e, ao mesmo tempo, servindo de orientação a novos testes (Schelini \& Wechsler, 2006).
O modelo de inteligência $\mathrm{CHC}$ tem se mostrado um dos mais abrangentes para a compreensão da inteligência humana, bem como para a sua avaliação (Alfonso, Flanagan \& Radwan, 2005; Kaufman, DeYoung, Gray, Brown \& Mackintosh, 2009; McGrew, \& Flanagan, 1998). Isso se deve ao fato de englobar diferentes capacidades humanas, de maneira hierárquica (Wechsler, Nakano, Nunes \& Minervino, 2010), tendo sido desenvolvido como uma extensão da teoria da inteligência fluida e cristalizada de Cattell a partir das contribuições de Horn e Carroll (Wasserman, 2012). A teoria é composta por dez tipos de habilidades gerais: Inteligência fluida (Gf); Inteligência cristalizada (Gc); 
Tendo-se como foco a avaliação da inteligência, o que se faz notar, no Brasil, é a inexistência, até o momento, de baterias disponíveis comercialmente baseadas nesse modelo teórico,

ainda que sua importância

venha sendo ressaltada

historicamente na literatura internacional, ainda que sejam encontrados estudos de validade com alguns instrumentos que tentam abranger um grande número de habilidades, podendo-se citar, como exemplos,

os estudos de Chiodi e Wechsler (2009), Schelini e Wechsler (2005).
Processamento visual (Gv); Processamento auditivo (Ga) Memória de curto prazo (Gsm); Recuperação de memória de longo prazo (Glr); Velocidade de processamento (Gs); Velocidade de decisão (Gt); Leitura e escrita (Gwr) e Conhecimento qualitativo (Gq), conforme apontado por Floyd, Keith, Taub e McGrew (2007), ainda que revisões mais recentes venham investigando a existência de novas habilidades (Táteis - Gh, Cinestésicas - Gk, Olfatórias - Go e Psicomotoras - GP), conforme exposto por Schneider e McGrew (2012).

Dada a complexidade do modelo, de acordo com Kaufman et al. (2009), a teoria CHC traz consigo uma evolução frente aos modelos de inteligência, principalmente porque integra as concepções de Cattell, Horn e Carroll, sem desprezar nenhum modelo, seja ele de Gf ou Gc, decompondo conceitos como a capacidade verbal em elementos mais simples como o desenvolvimento da linguagem, o conhecimento léxico, a capacidade de informação geral, a capacidade de comunicação e a sensibilidade gramatical, de modo a facilitar a visualização e compreensão do que deve ser avaliado. Por esse motivo, McGrew (2009) infere que o modelo CHC é o que possui as melhores evidências psicométricas para descrever as aptidões humanas, de maneira a ser considerado, segundo apontamento de Schelini e Wechsler (2006) como o modelo mais adequado para a construção de instrumentos psicológicos para a avaliação da inteligência.

Tendo-se como foco a avaliação da inteligência, o que se faz notar, no Brasil, é a inexistência, até o momento, de baterias disponíveis comercialmente baseadas nesse modelo teórico, ainda que sua importância venha sendo ressaltada historicamente na literatura internacional, ainda que sejam encontrados estudos de validade com alguns instrumentos que tentam abranger um grande número de habilidades, podendo-se citar, como exemplos, os estudos de Chiodi e Wechsler (2009), Schelini e Wechsler (2005). A situação fica ainda mais complicada ao se pensar na avaliação da inteligência de crianças, ainda que, internacionalmente, uma grande diversidade de baterias seja encontrada para essa faixa etária, conforme levantamento realizado por Ford, Kozey e Negreiros (2012). O mesmo quadro é encontrado quando se aborda a investigação desse construto em populações minoritárias, podendo-se encontrar uma situação bastante diferente fora do país, tais como os exemplos de estudos desenvolvidos com indivíduos com dificuldades de aprendizagem (Flanagan, Alfonso, Mascolo \& Sotelo-Dynega, 2012), autismo (Klinger, O'Kelley, Mussey, Goldstein \& DeVries, 2012), transtorno de atenção e hiperatividade (Hale, Yim, Schneider, Wilcox, Henzel \& Dixon, 2012), deficiência sensorial (Illas, Barada, Pérez \& González, 2003) e física (Decker, Englund \& Roberts, 2012) e deficiência intelectual (Armstrong, Hangauer \& Nadeau, 2012).

Especificamente em relação à avaliação de crianças deficientes visuais, na literatura científica nacional pode ser observada uma escassez de pesquisas que fizeram uso de testes psicológicos na avaliação dessa população (Nascimento \& Flores-Mendoza, 2007; Nicolaiewsky \& Correa, 2009), assim como a inexistência, no Brasil, de instrumentos psicológicos válidos e padronizados especificamente para uso nessa população especial. De acordo com Masini (1995), os testes que vêm sendo utilizados no Brasil mostram-se ainda bastante restritos, ainda que voltados à investigação de diversos construtos. Como exemplo, pode ser citado o teste Ohwaki Kohs, o qual envolve cubos táteis destinados para avaliação da inteligência a partir dos seis anos de idade; o Teste de Inteligência Williams, para crianças com cegueira e visão subnormal até 12 anos que tem como foco a avaliação com base nas atitudes e rendimentos; e a Escala Ballard e Barraga que é de origem americana, mas que foi adaptado para a população brasileira (Lobato, 2005). Esses testes visam avaliar o nível de funcionamento das habilidades das crianças com deficiência visual, podendo ser medida a capacidade de associação, memória 
imediata, pensamento lógico, atenção, imitação, generalização, raciocínio indutivo e raciocínio dedutivo, psicomotricidade, entre outras (Masini, 1995). A Escala Ballard e Barraga auxilia a verificação do problema visual em termos educacionais por meio da apresentação de objetos cada vez menores, como a Escala Snellen, determinando a eficiência do uso da visão por parte da criança (Kirk \& Gallagher, 2002), devendo-se ressaltar o fato de que nenhum desses encontra-se traduzido, validado e disponível para uso no Brasil.

Segundo Brambring e Troster (1994), essa falta de instrumentação traz como consequência a falta de investigações e estudos sobre o desenvolvimento e perfil cognitivo de crianças e adultos com essa peculiaridade. Embora a literatura nacional tenha estudos importantes (Machado, 2011; Nunes \& Lomônaco, 2008; Rabello, Motti \& Gasparetto, 2007), a produção científica internacional tem se mostrado bastante avançada na avaliação de portadores de deficiências, podendo-se citar, como exemplo, o manual americano do WAIS-III, o qual apresenta estudos com grupos especiais, tendo sido traduzido para atender à população surda por meio da linguagem americana de sinais (Nascimento \& FloresMendoza, 2007).

Essa escassez de material especializado pode ser justificada pela dificuldade de adaptação e padronização exigidas para se formalizar um teste. Isso porque, segundo Baron (2006), todo teste deve ter condições de ser aplicado em qualquer público, devendo o mesmo ser adaptado sem que seu objetivo seja alterado (ou seja, continue a avaliar o que pretende) e estar de acordo com os recursos necessários que o participante necessita sem que nada prejudique o seu desempenho. Entretanto, não se pode deixar de considerar que "a deficiência visual impõe restrições às capacidades de movimento livre, seguro e confiante da criança no ambiente" (Malta, Endriss, Rached, Moura \& Ventura, 2006, p.571), de forma que um planejamento antecedente à aplicação do teste mostra-se necessário para obter informações acerca da melhor forma de aplicação e execução da atividade (com presença de luz/ sem presença de luz/ através de outros sentidos - tátil, auditivo, olfativo) (Bizerra, Cizauskas, Inglez \& Franco, 2012; Lara, 2009; Pereira, 2009), dada a importância de se considerar o tipo e o grau de deficiência, bem como a funcionalidade da visão residual, que pode ser diferente para cada individuo como relatam Joyce, Isom, Dial e Sandel, (2004) e Montilha, Gasparetto e Nobres, M (2002). Tal cuidado visa garantir que o participante tenha disponível todos os recursos que substituam a visão para que o resultado no teste não se torne inválido.

Segundo orientações da American Educational Research Association, American Psychological Association e National Council on Measurement in Education (1999), cuidados devem ser tomados na testagem de indivíduos com deficiências, incluindo-se modificações no formato de apresentação do teste ou das respostas, tempo disponível, ambiente, uso de partes do instrumento ou emprego de testes alternativos, quando possível. Nesse mesmo sentido, Decker et al. (2012) atribuem ao psicólogo a responsabilidade na seleção de instrumentos apropriados, de modo a evitar viés na administração ou interpretação dos resultados, de modo a considerar as modificações necessárias para cada criança. Tal constatação justifica a importância de um processo diferenciado de avaliação da inteligência de deficientes visuais, visto que a visão desempenha um papel muito importante no começo da vida, pois é uma ferramenta estimuladora para a comunicação e a realização de tarefas (Kirk \& Gallagher 2002), podendo trazer dificuldades ao desenvolvimento tanto cognitivo como social de uma criança, influenciando também em seu rendimento físico e intelectual, conforme apontado por Monteiro e Montilha (2010). O problema situa-se no fato de que, de acordo com Chiodi e Wechsler (2009), falta, na realidade brasileira, instrumentos validados que permitam conhecimentos a respeito da competência e habilidade de crianças, em especial daquelas com algum tipo de deficiência, de maneira que 
...considerando as diversas

evidências e avaliações sobre o processo de desenvolvimento e de qualidade de vida das pessoas cegas, deve-se enfatizar que os resultados obtidos até o momento apontam para a importância de se trabalhar a favor da deficiência considerandose uma ampla variedade de situações, dada a oportunidade desses indivíduos de desenvolver, de forma normal, diversas outras habilidades e capacidades (Roberts, 1996). essa realidade acaba reduzindo informações que auxiliariam no diagnóstico e na intervenção junto a esses sujeitos.

Portanto, considerando as diversas evidências e avaliações sobre o processo de desenvolvimento e de qualidade de vida das pessoas cegas, deve-se enfatizar que os resultados obtidos até o momento apontam para a importância de se trabalhar a favor da deficiência considerandose uma ampla variedade de situações, dada a oportunidade desses indivíduos de desenvolver, de forma normal, diversas outras habilidades e capacidades (Roberts, 1996). Nessa perspectiva, esses dados contribuem com o objetivo central dos testes psicológicos que avaliam o desenvolvimento cognitivo, pois possibilitam que, embora o indivíduo não disponha do recurso visual, seus outros sentidos possam ser bem desenvolvidos, facilitando sua interação com o mundo.

Diante do exposto, e tendo-se como foco a avaliação das habilidades cognitivas de crianças com deficiência visual, o objetivo deste estudo foi construir subtestes para avaliação desse construto nessas crianças, bem como a realização de estudo-piloto a fim de verificar sua adequação para a população alvo.

\section{Método}

\section{Estudo 1 \\ Participantes}

A amostra deste estudo foi composta por 14 crianças deficientes visuais, na faixa etária de 7 a 12 anos $(M=10,28$ anos; $D P=1,58)$, sendo seis do sexo feminino e oito do sexo masculino. A amostra foi caracterizada por classificação de tipo e grau de deficiência visual, assim sendo, dez crianças que responderam aos testes eram classificadas com baixa visão, sendo oito com deficiência congênita e duas com doença adquirida e quatro crianças classificadas com cegueira total, sendo duas com deficiência adquirida e duas com deficiência congênita. Quanto à escolaridade, uma criança cursava o segundo ano do ensino fundamental, três crianças cursavam o quarto ano, cinco cursavam o quinto ano, quatro cursavam o sexto ano e uma cursava o oitavo ano.

\section{Instrumento}

\section{Subteste 1 - Inteligência Verbal (Analogias)}

Busca avaliar a capacidade da criança na identificação de analogias verbais, sua compreensão de associação e seu conhecimento verbal. É composto por três exemplos e 25 itens, nos quais a criança deve completar a frase com a palavra correta que é anotada na folha de resposta. A criação dos itens foi baseada principalmente tomandose como base o tipo de tarefa presente no subteste de raciocínio verbal da Bateria de Provas de Raciocínio (Primi \& Almeida, 2000), em suas formas A e B e na sua versão infantil. Deve-se salientar que se optou pela realização oral do teste, sem tempo limite (devido ao fato desse ser um estudo exploratório) embora o mesmo tenha sido controlado, a fim de observar o tempo médio de duração para realização do subteste. Nesta atividade, a proposta de correção baseia-se na ideia de fornecer um ponto para cada resposta correta.

\section{Subteste 2 - Memória}

Pretende avaliar a capacidade de memória a curto prazo da criança por meio de um jogo tradicional de memória, com a diferença de que as peças, ao invés de figuras, possuem texturas, de modo a poderem ser identificadas por meio do tato, e não da visão. É composto por 13 pares de peças texturizadas em apenas um de seus lados, tendo sido desenvolvido tomando-se como base um jogo de texturas utilizado em uma das instituições que atendem as crianças que participaram da pesquisa. 
Nesta atividade, optou-se pela contagem do número de pares abertos pela criança, independentemente dela acertá-lo ou não. Da mesma forma, o número de acertos também foi pontuado, contando-se também o número de erros cometidos e o tempo de execução da atividade.

\section{Subteste 3 -}

\section{Pensamento Lógico-Espacial}

Busca avaliar a capacidade da criança em estabelecer relações por meio de duas sequências lógicas contidas nas figuras. Nele, a criança deve compreender qual modificação foi realizada na primeira relação para aplicar o mesmo princípio na segunda relação, de modo a encontrar a resposta correta. O subteste é composto de três exemplos e 18 itens, montados, individualmente, em folha de E.V.A. tamanho A2. As figuras foram construídas pensando-se em diferentes níveis de dificuldade, a partir do número de critérios envolvidos em cada item, os quais podiam relacionar-se à modificação da forma (círculo, quadrado e triangulo), tamanho (pequeno ou grande) e textura (presente ou ausente), sendo os itens fáceis aqueles com a alteração de um dos critérios, os itens de dificuldade média envolviam a alteração conjunta de dois critérios e os itens difíceis envolviam três critérios, em um total de seis itens para cada nível de dificuldade. Deve-se salientar ainda que, nesta atividade, optou-se por pontuar um ponto para cada resposta correta. Assim sendo, a criança responde indicando uma das alternativas de múltipla escolha (dentre cinco fornecidas), sendo o tempo de execução também controlado.

\section{Procedimentos}

Para a construção dos subtestes tomouse como referência os modelos teóricos mais atuais que abarcam o construto, bem como os testes de avaliação da inteligência comercializados e aprovados pelo Sistema de Avaliação de Testes Psicológicos (Satepsi).
Para torná-los específicos e direcionados para avaliação de crianças com deficiência visual, entrevistas livres com profissionais especializados foram realizadas, a fim de coletar informações sobre metodologias, materiais e instrumentos utilizados em instituições de atendimento a deficientes visuais. Posteriormente, o processo de construção baseou-se nessas informações, com a preocupação de priorizar materiais da vida diária dessas crianças. Posterior às etapas de construção, a coleta de dados deste estudo foi iniciada após aprovação da pesquisa pelo Comitê de Ética da Pontifícia Universidade Católica de Campinas (PUC-Campinas), sob número 01082812.6.0000.5481.

Assim, considerando o subteste 1 Inteligência Verbal (Analogias), deve-se inferir que inicialmente foi composto por dezoito itens, que passaram por um pré-teste em duas crianças com visão normal (uma sexo feminino com oito anos de idade e uma do sexo masculino com doze anos de idade) a fim de verificar possíveis dificuldades quanto à execução e compreensão da atividade e entendimento das frases construídas.

Não foi notada nenhuma dificuldade quanto à compreensão das instruções fornecidas, sendo que a primeira criança acertou 16 itens em um tempo de $12^{\prime} 18^{\prime \prime}$ e a segunda criança acertou os 18 itens em 8'10'. Devido à quantidade de acerto para ambas as crianças, a pesquisadora optou por construir mais sete itens, envolvendo conceitos mais abstratos, na tentativa de aumentar a dificuldade dos itens, de modo que, a versão a ser testada acabou por ser composta por 25 itens.

Quanto ao subteste 2 Memória, para a confecção do material foram utilizados materiais de fácil acesso. Assim sendo, a primeira confecção do jogo de memória continha 18 pares de texturas. O material foi testado nas mesmas crianças, as quais, durante a aplicação, utilizaram uma venda nos olhos. A partir dessa aplicação foi possível perceber a dificuldade de identificar texturas parecidas (por exemplo: tecido de algodão/ 
feltro e papel camurça) e fazer pares, sendo estes confundidos nas duas aplicações. Dessa forma, optou-se por retirar os pares de tecido e de papel camurça, visto que o objetivo do teste não é o de identificar texturas e sim, memorizar suas localizações.

Ainda quanto às texturas, dois outros pares foram retirados do jogo, um deles que continha o lado amarelo de uma esponja comum (liso) e o outro que continha o lado verde da esponja (áspero). Esses pares foram retirados devido à facilidade de encontrálos quando exposto à criança, uma vez que quando permaneciam com a textura para baixo, as peças ficavam mais altas do que as demais, facilitando encontrá-las. Quanto à condução das instruções, as crianças não tiveram dificuldades no entendimento, tampouco apresentaram sinais de fadiga. Deve-se salientar ainda que o tempo de execução da atividade nestas aplicações foi contado, sendo a atividade realizada pela criança do sexo feminino em 10'56" e pela criança do sexo masculino em 9'58'. Ambos acertaram as 13 peças que compõem o teste. Finalizando, o subteste 3 Lógico-Espacial também passou pela pré-testagem. As crianças testadas demonstraram dificuldades nos três últimos itens, os quais são considerados mais difíceis. No entanto, nenhum sinal de fadiga quanto à realização do subteste foi observado ou relatado.

A primeira criança testada respondeu a todos os itens em um tempo de $17^{\prime} 17^{\prime \prime}$, acertando 16 itens, tendo errado 2 itens, os quais depois observou os erros causados pela mudança de critério de textura em um item e de tamanho no outro. Por outro lado, a segunda criança respondeu aos 18 itens em 8'19"e acertou 17 itens, errando um pela mudança de textura. Os participantes relataram gostar da atividade, ainda que este tenha sido mais demorado e difícil. Considerando nenhuma dificuldade aparente pelos participantes que realizaram o pré-teste, o subteste Pensamento Lógico-Espacial não sofreu modificação quanto à estrutura dos itens ou mesmo da instrução para a versão a ser testada com a amostra de deficientes visuais.

Assim, posterior às alterações realizadas, iniciou-se o processo de aplicação na amostra de crianças deficientes visuais. Dessa forma, após o consentimento da instituição, as crianças foram selecionadas a partir da indicação dos profissionais e do levantamento do tipo/classificação da cegueira. Após a permissão dos responsáveis, a aplicação ocorreu individualmente. Alguns dados foram levantados no dia da aplicação, com o objetivo de identificar o tipo e o grau de deficiência do participante, bem como conhecer suas necessidades específicas e explicar os procedimentos e objetivo do estudo. As atividades tiveram duração aproximada de duas horas.

Deve-se ressaltar, aqui, que dois participantes não responderam ao subteste Lógico-Espacial por motivos de saúde, fato que poderia influenciar no desempenho das atividades, tendo-se decidido pela manutenção dos resultados dos mesmos durante a análise dos dois outros subtestes.

\section{Resultados}

Com o objetivo de testar a adequação do instrumento e visando identificar possíveis diferenças de desempenho na capacidade intelectual, de acordo com o tipo de deficiência visual da criança (congênita $x$ adquirida, cegueira total $x$ baixa visão), para cada medida dos subtestes (verbal $=$ tempo de execução e total de acertos; memória = tempo de execução, total de acertos e número de peças abertas; espacial = tempo de execução e total de acertos), a primeira análise consistiu na realização do teste não paramétrico Mann-Whitney, comparando o desempenho dos grupos em relação ao tipo de deficiência (congênita $x$ adquirida) apresentada na Tabela 1 e grau de deficiência (cegueira $x$ baixa visão), cujos resultados são apresentados na Tabela 2. 
Tabela 1. Estatística descritiva e teste de diferença de médias para tipo de deficiência (congênita $\mathrm{x}$ adquirida) nos resultados dos três subtestes

\begin{tabular}{|c|c|c|c|c|c|c|}
\hline Subteste & Medida & $\begin{array}{l}\text { Tipo de } \\
\text { deficiência }\end{array}$ & Média & D.P. & $U$ & Sign. \\
\hline \multirow{4}{*}{ Verbal } & \multirow[t]{2}{*}{ Tempo de execução (em minutos) } & Adquirida & 6,58 & 2,22 & \multirow[t]{2}{*}{13,0} & \multirow[t]{2}{*}{0,322} \\
\hline & & Congênita & 8,48 & 3,57 & & \\
\hline & \multirow[t]{2}{*}{ Total de acertos } & Adquirida & 13,00 & 4,54 & \multirow[t]{2}{*}{18,0} & \multirow[t]{2}{*}{0,777} \\
\hline & & Congênita & 13,10 & 5,78 & & \\
\hline \multirow{4}{*}{ Memória } & \multirow[t]{2}{*}{ Tempo de execução (em minutos) } & Adquirida & 7,23 & 5,48 & \multirow[t]{2}{*}{16,0} & \multirow[t]{2}{*}{0,572} \\
\hline & & Congênita & 5,16 & 2,13 & & \\
\hline & \multirow[t]{2}{*}{ Total de acertos } & Adquirida & 11,00 & 2,82 & \multirow[t]{2}{*}{11,0} & \multirow[t]{2}{*}{0,077} \\
\hline & & Congênita & 12,90 & 0,31 & & \\
\hline & \multirow[t]{2}{*}{ Total de aberturas } & Adquirida & 84,00 & 50,88 & \multirow[t]{2}{*}{13,5} & \multirow[t]{2}{*}{0,357} \\
\hline \multirow{5}{*}{ Espacial } & & Congênita & 58,80 & 17,10 & & \\
\hline & \multirow[t]{2}{*}{ Tempo de execução (em minutos) } & Adquirida & 26,42 & 24,26 & \multirow[t]{2}{*}{6,0} & \multirow[t]{2}{*}{0,390} \\
\hline & & Congênita & 16,10 & 9,07 & & \\
\hline & \multirow[t]{2}{*}{ Total de acertos } & Adquirida & 2,00 & 2,30 & \multirow[t]{2}{*}{3,0} & \multirow[t]{2}{*}{$0,015^{*}$} \\
\hline & & Congênita & 7,50 & 3,10 & & \\
\hline
\end{tabular}

De acordo com a Tabela 1, nota-se que foram encontrados resultados significativos apenas no subteste Lógico-Espacial, em relação ao tipo de deficiência, sendo possível visualizar que as crianças com deficiência congênita obtiveram melhor desempenho $(M=7,50)$, em média de acertos em comparação às crianças com deficiência adquirida $(M=2,00)$, sendo a diferença significativa $(U=3 ; z=-2,42 ; p=0,015)$. Nas demais medidas dos subtestes, as diferenças de média não se mostraram significativas.

Deve-se atentar que os dados distribuídos para cada classificação (congênita x adquirida), referemse a uma amostra reduzida de crianças, sendo dez que compõem a amostra de crianças com deficiência congênita e quatro que compõem a amostra de crianças com deficiência adquirida. Assim sendo, deve-se considerar essa limitação durante a compreensão dos resultados descritos.

Após a análise dos participantes em relação ao tipo de deficiência, uma segunda análise visou comparar os resultados obtidos pelos participantes quanto ao grau de deficiência (cegueira $x$ baixa visão). Novamente, o teste não paramétrico de Mann Whitney foi utilizado pelos mesmos motivos apontados anteriormente. A Tabela 2 traz os resultados dos participantes quanto ao grau de deficiência, comparando seu desempenho nas medidas dos três subtestes. 
Tabela 2. Estatística descritiva e teste de diferença de médias para grau de deficiência nos resultados dos três subtestes

\begin{tabular}{lllllll}
\hline Subtestes & Medida & Grau de & Média & DP & U & Sign. \\
& deficiência & & & & \\
\hline \multirow{2}{*}{ Verbal } & Tempo de execução & Baixa Visão & 14,00 & 5,56 & 19,0 & 0,888 \\
& (em minutos) & Cegueira & & 12,15 & 4,99 & \\
& Total de acertos & Baixa Visão & 8,12 & 4,19 & 16,5 & 0,620 \\
& Cegueira & & 7,49 & 2,17 & \\
& Tempo de execução & & & & & \\
& (em minutos) & Baixa Visão & 4,23 & 2,14 & 4,0 & $0,024^{*}$ \\
& & Cegueira & & 8,53 & 4,25 & \\
Memória & Total de acertos & Baixa Visão & 12,90 & 0,31 & 11,0 & 0,077 \\
& & Cegueira & & 11,00 & 2,82 & \\
& Total de aberturas & Baixa Visão & 54,20 & 15,12 & 4,0 & $0,023 *$ \\
& & Cegueira & & 95,50 & 41,96 & \\
& Tempo de execução & Baixa Visão & 13,33 & 7,16 & 4,0 & 0,079 \\
Lógico- & (em minutos) & Cegueira & & 30,35 & 16,07 & \\
Espacial & Total de acertos & Baixa Visão & 6,00 & 3,71 & 19,5 & 0,943 \\
& & Cegueira & & 5,75 & 4,64 & \\
\hline & & & & &
\end{tabular}

Os resultados demonstraram resultados significativos apenas no subteste de Memória, na medida do tempo gasto na execução da tarefa, visto que crianças com cegueira demoraram mais tempo para a realização da atividade $\left(8^{\prime} 53^{\prime \prime}\right)$ do que as crianças com baixa visão $\left(4^{\prime} 23^{\prime \prime}\right)$, sendo $U=4$; $z=-2,26 ; p=0,024$. No mesmo subteste, também foram encontradas diferenças significativas quanto à quantidade de abertura de peças $(U=4 ; z=-2,26 ; p=0,023)$, sendo que as crianças com baixa visão abriram menos pares de peças $(M=54,20)$ do que as crianças com cegueira $(M=95,50)$. Nas demais medidas e nos demais subtestes, nenhuma diferença significativa foi encontrada em relação ao grau de deficiência.

\section{Discussão}

É interessante mencionar que o presente estudo considerou a importância de um processo diferenciado de avaliação da inteligência para deficientes visuais, uma vez que a visão desempenha um papel muito importante no começo da vida, sendo uma ferramenta estimuladora para a comunicação e a realização de tarefas. Dessa forma, considerou o fato de que, como aponta Monteiro e Montilha (2010), qualquer tipo de deficiência, sendo ela total ou parcial, pode influenciar diretamente o desenvolvimento cognitivo e social de uma criança, assim como seu rendimento físico e intelectual. Por tal motivo, as atividades propostas em cada subteste foram especialmente pensadas e planejadas de modo a se tentar atender às necessidades especiais da população-alvo do estudo, fazendo-se uso de materiais, recursos e cuidados diferenciados daqueles que usualmente são utilizados nos testes de avaliação da inteligência.

Isso porque, conforme afirma Roberts (1996), mesmo sem o recurso da visão, as crianças com deficiência tendem a trabalhar a favor dela ao se defrontarem com uma variedade de 
Pode-se

dizer que, de forma geral, os resultados evidenciaram que as crianças com deficiência congênita obtiveram melhores resultados que as crianças com deficiência adquirida, fato que pode ser justificado a partir da concepção exposta por Nunes e Lomônaco (2008). situações, podendo desenvolver outros tipos de habilidades. Opinião similar é apresentada por Bizerra et al. (2012) ao fazerem menção à importância da estimulação e desenvolvimento de outros sentidos. De acordo com os autores, essa estimulação facilita o processo de significação de objetos e conhecimento, fato que foi possível observar também durante as aplicações. No subteste Memória, por exemplo, as crianças cegas fizeram uso do recurso tátil na maioria das vezes, salvo casos de crianças que desenvolveram táticas diferentes de identificação da peça, por meio, por exemplo, do recurso auditivo (batendo as peças na mesa para identificar sons iguais provenientes de peças que formavam pares) $\mathrm{e}$ do recuso olfativo, sentindo o cheiro que cada peça possuía e identificando cheiros comuns. No subteste Lógico-Espacial, também fizeram uso de recursos alternativos como, por exemplo, da audição para identificar a presença/ausência de textura nas peças e do tato para o tamanho e mudança de forma. Tal constatação reforça a percepção de Nunes e Lomônaco (2010), assim como Pereira (2009), segundo os quais o tato assume papel fundamental no conhecimento de deficientes visuais, visto que é por meio dele que a criança é capaz de reconhecer tamanhos, texturas, formas e temperaturas, uma vez que ela passa a apresentar esse conhecimento a partir do momento que entra em contato com o objeto, percorre determinada distância ou sente alguma temperatura na pele.

Quanto ao subteste Verbal, foi possível observar que as crianças deficientes mostraram bom desempenho, o qual pode ser explicado diante da ênfase que vem sendo dada por pesquisadores da área ao apontarem que o recurso mais utilizado por crianças deficientes visuais é a linguagem oral falada (Baron, 2006). No entanto, de acordo com a autora, alguns cuidados devem ser considerados quando se utiliza desse recurso, como ambiente silencioso, nenhum tipo de interrupção ou barulho, a dicção do aplicador, tom de voz alto e fala limpa. Deve-se inferir, aqui, que a pesquisadora levou esses aspectos em consideração a cada aplicação realizada a fim de proporcionar o melhor ambiente de aplicação possível.

Pode-se dizer que, de forma geral, os resultados evidenciaram que as crianças com deficiência congênita obtiveram melhores resultados que as crianças com deficiência adquirida, fato que pode ser justificado a partir da concepção exposta por Nunes e Lomônaco (2008). De acordo com os autores, as crianças com deficiência congênita tendem a incorporar conceitos por meio de conceitos ou significados de outras palavras ou objetos que já tenham tido experiência anterior, ou seja, devido ao fato de nunca terem provido do recurso da visão, tendem a utilizarem de outras formas de aprendizado para resgatar informações, tendo assim maior domínio dos conceitos aprendidos e tendendo a estar mais familiarizadas com a atividade. Ainda nessa perspectiva, o estudo de Illas et al. (2003) também afirma que a perda da visão vem comumente associada à perda da independência quanto a sua mobilidade, capacidade de leitura e escrita, e independência pode desencadear desajustes emocionais e mentais, necessitando que a criança tenha que aprender a utilizar de outros recursos para conhecer o mundo, diferentemente da criança com deficiência congênita, a qual o mundo já foi construído sem o recurso visual.

Isso porque, de acordo com Decker et al. (2012), a idade em que a perda da visão se deu é uma informação de extrema importância, visto que contribui para a heterogeneidade dessa população. Segundo os autores, resultados de estudo demonstraram que adultos que haviam perdido a visão antes dos 17 anos apresentaram desempenho superior do que aqueles que haviam perdido a visão com mais idade, principalmente em relação 
à discriminação de formas e texturas, de modo a evidenciar a influência da idade em que a deficiência é desenvolvida e seu impacto nos resultados dos testes. Da mesma forma, tal resultado foi obtido no presente estudo ao se verificar melhor desempenho das crianças com deficiência congênita quando comparadas àquelas com deficiência adquirida.

Os mesmos autores também ressaltam que outra variável que pode afetar a performance nos instrumentos refere-se à cegueira ou quantidade de visão perdida, ou seja, o grau da deficiência, investigado no presente estudo. Isso porque o primeiro caso, o mais severo grau, exigirá modificações tanto na testagem, no conteúdo e nos métodos utilizados, ao passo que o segundo caso (cegueira parcial) poderá, em muitos casos, exigir somente adequações relacionadas ao tamanho da impressão do material. Tal constatação foi confirmada em estudo desenvolvido por Joyce et al. (2004), no qual participantes com visão residual obtiveram melhor desempenho em baterias de inteligência do que aqueles com cegueira total, de modo a demonstrar a importância de se considerar o nível de visão perdida durante a avaliação de indivíduos com deficiência visual. Novamente, os resultados obtidos com os subtestes construídos neste estudo também apontaram para melhor desempenho das crianças com visão residual quando comparadas àquelas com cegueira total.

Especificamente em relação à avaliação das habilidades cognitivas, esta pesquisa levou em consideração os apontamentos que vêm sendo destacados pela literatura internacional espanhola, a qual traz contribuições e avanços consideráveis frente aos estudos envolvendo cegos e com baixa visão. Segundo Lara (2009), é notada e entendida a preocupação em atender às necessidades específicas de cada criança, bem como o uso de recursos táteis e de forma verbal. Da mesma forma, para as crianças que possuem algum tipo de visão residual, reforçar a utilização desse tipo de recurso mostra-se de extrema relevância. Interessante mencionar também que a visão residual para as crianças com baixa visão foi notada nesta pesquisa como relevante para o desempenho das mesmas quando comparadas com as crianças cegas, visto que tais crianças obtiveram desempenhos melhores nos subtestes. Nesse sentido, estudo de Montilha et al. (2002) reforça a percepção de que a criança com baixa visão faz uso da visão residual durante a realização de atividades, uma vez que a estimulação desse tipo de visão mostrou aumentar a possibilidade de um melhor desempenho.

\section{Considerações finais}

Considerando o objetivo de construir uma bateria de subtestes para a avaliação da inteligência de crianças com idades entre sete a 12 anos, com deficiência adquirida ou congênita e seus diferentes tipos (cegueira total ou baixa visão), os resultados apontaram para o fato de que os subtestes criados atenderam às necessidades dessa população específica, mostrando-se, em um primeiro momento, adequados para a avaliação da inteligência de crianças com cegueira e baixa visão, bem como a importância de se respeitar as diferenças e particularidades dessa população.

Quanto ao instrumento, pode-se dizer que sua construção foi percebida como de extrema importância, uma vez que a escassez de materiais na temática é algo que tem gerado bastante preocupação por parte de profissionais e pesquisadores da área. Assim sendo, o desenvolvimento de um instrumento psicológico capaz de avaliar habilidades cognitivas de crianças com deficiência visual mostra-se bastante relevante para a área da avaliação psicológica no país. No entanto, deve-se ressaltar o caráter ainda 
exploratório deste estudo, assim como o fato de que tal instrumento, ao longo de todo o processo que ainda deve ser seguido até sua disponibilização para uso profissional por meio da publicação, deverá passar por importantes reformulações e adequações, as quais lhe possibilitarão melhor qualidade. Os dados obtidos nos estudos aqui relatados e a observação de que os subtestes criados mostraram-se, em um primeiro momento, adequados para uso na população-alvo, incentivam a condução de novos estudos, podendo-se citar, dentre eles, aqueles que visam a investigação dos parâmetros psicométricos, especialmente a busca de evidências favoráveis de validade e precisão.

Acredita-se que o presente estudo, de caráter exploratório, trouxe contribuições relevantes dentro da área da avaliação psicológica. No entanto, deve-se inferir que a pesquisa traz limitações, principalmente em relação ao número reduzido de participantes, devido à dificuldade em se localizar crianças com esse quadro ou instituições de atendimento às mesmas. Dessa forma, estudos com amostras maiores são recomendados a fim de que o instrumento criado possa ser investigado em relação às exigências impostas pelo Conselho Federal de Psicologia (CFP) para sua disponibilização, de modo a trazer novas perspectivas sobre a temática.

Em relação aos resultados mencionados, pode-se dizer que estes trouxeram informações importantes quanto à construção dos subtestes, bem como quanto às diferenças de cada tipo e grau de deficiência, fato que poderá ser melhor investigado em estudos futuros sendo que, caso tais resultados sejam confirmados, justificariam a necessidade de construção de tabelas especificas para a avaliação da criança de acordo com seu diagnóstico de deficiência visual, em alguns subtestes e referente a algumas medidas, como tempo de execução, quantidade de acertos, entre outros já mencionados. Assim, reforça-se a importância de que, não só se faça notar as diferentes necessidades e particularidades entre crianças videntes e deficientes visuais, mas também, dentro desse último rótulo, a necessidade de se respeitar o tipo e o grau. 


\section{Carolina Rosa Campos}

Doutoranda em Psicologia na Pontifícia Universidade Católica de Campinas, Campinas - SP - Brasil.

E-mail: carolene_crc@hotmail.com

\section{Tatiana de Cássia Nakano}

Doutora em Psicologia como Profissão e Ciência pela Pontifícia Universidade Católica de Campinas. Docente do curso de pós-graduação Stricto Sensu em Psicologia da Pontifícia Universidade Católica de Campinas, Campinas - SP - Brasil. E-mail: tatiananakano@hotmail.com

\section{Endereço para envio de correspondência:}

Rua Ferreira Penteado, 960, apto 2B. CEP: 13010-041. Campinas, SP.

Recebido 18/12/2012, 1a Reformulação 16/09/2013, Aprovado 16/10/2013.

\section{Referências}

Alfonso, V.C., Flanagan, D.P. \& Radwan, S. (2005).The Impact of the Cattell-Horn-Carroll Theory on Test development and Interpretation of Cognitive and Academic Abilities. In D. P. Flanagan \& P. L. Harrison (Orgs.), Contemporary Intellectual Assessment (2a ed., pp 185-202). New York: Guilford.

Almeida, L.S., Guisande, AM.A., Primi, R. \& Ferreira, A. (2008). Construto e medida da inteligência: contributos da abordagem fatorial. In A. Candeias, L.S. Almeida, A. Roazzi \& R. Primi (Orgs.), Inteligência: definição e medida na confluência de múltiplas concepções (pp.49-80). São Paulo: Casa do Psicólogo.

American Educational Association, American Psychological Association, National Council on Measurement in Education (1999). Standards for educational \& psychological tests. Washington, DC: American Psychological Association.

Armstrong, K., Hangauer, J. \& Nadeau, J. (2012). Use of intelligence tests in the identification of children with intellectual and developmental disabilities. In D.P. Flanagan \& P.L. Harrison (Orgs.), Contemporary intellectual assessment (pp. 726-737). New York: The Guilford Press.

Baron, H. (2006). Visual Impairment and psychometric testingPractical advice for test users managing the testing of people who have sight disabilities. Recuperado de http:// www. psyasia.com, VisuallmpairmentandPsychologicalTesting.pdf.

Bizerra, A.F., Cizauskas, J.B.V., Inglez, G.C., \& Franco, M.T. (2012). Conversas de aprendizagem em museus de ciências: como os deficientes visuais interpretam os materiais educativos do museu de microbiologia. Revista Educação Especial (Santa Maria), 25(42), 57-74.

Brambring, M., \& Troster, H. (1994). The assessment of cognitive development in blind infants and preschoolers. Journal of Visual Impairment \& Blindness, 88(1), 9-18.
Chiodi G.M., \& Wechsler M. S. (2009). Escala de Inteligência WISC-III e Bateria de Habilidades Cognitivas Woodcock Johnson-III: comparação de instrumentos. Avaliação Psicológica; 8(3), 313-324.

Decker, S.T., Englund, J.A., \& Roberts, A.M. (2012). Intellectual and neuropsychological assessment of individuals with sensory and physical disabilities and traumatic brain injury. In D.P. Flanagan \& P.L. Harrison (Orgs.), Contemporary intellectual assessment (pp. 708-725). New York: The Guilford Press.

Flanagan, D.P., Alfonso, V.C., Mascolo, J.T., \& Sotelo-Dynega, M. (2012). Use of ability tests in the identification of specific learning disabilities within the context of an operational definition. In D.P. Flanagan \& P.L. Harrison (Orgs.), Contemporary intellectual assessment (pp. 643-669). New York: The Guilford Press.

Floyd, R.G., Keith, T.Z., Taub, G.E., \& McGrew, G.E. (2007) Cattell-Horn-Carroll Cognitive Abilities and Their Effects on Reading decoding Skills: $g$ Has Indirect Effects, More Specific Abilities Have Direct Effects. School Psychology Quarterly, 22(2), 200-233. doi: 10.1037/1045-3830.22.2.200

Ford, L., Kozey, M.L., \& Negreiros, J. (2012). Cognitive assessment in early childhood: theoretical and practice perspectives. In D.P. Flanagan \& P.L. Harrison (Orgs.), Contemporary intellectual assessment (pp. 585-622). New York: The Guilford Press.

Hale, J.B., Yim, M., Schneider, A.N., Wilcox, G., Henzel, J.N. \& Dixon, S.G. (2012). Cognitive and neuropsychological assessment of attention-deficit/hyperactivity disorder: redefining a disruptive behavior disorder. In D.P. Flanagan \& P.L. Harrison (Orgs.), Contemporary intellectual assessment (pp. 687-707). New York: The Guilford Press. 
Illas, L.O., Barada, D.L.H., Pérez, J.A.P., \& González, C.P. (2003). Prevalência de baja visión y ceguera em um área de salud. Revista Cubana de Medicina Ceneral Integral, 19(5), 1-8.

Joyce, A., Isom, R., Dial, J.G., \& Sandel, M.H. (2004). Implications of perceptualmotor differences within blind populations. Journal of Applied Rehabilitation Counseling, 35(3), 3-7.

Kaufman, S. B., DeYoung, C. G., Gray, J. R., Brown, J., \& Mackintosh, N. (2009). Associative learning predicts intelligence above and beyond working memory and processing speed. Intelligence, 37(4), 374-382. doi: 10.1016/j.intell.2009.03.004

Kirk, S. A., Gallagher, J. J. (2002) Crianças com deficiência visual. In S. A. Kirk, \& J.J. Gallagher. Educação da Criança Excepcional (pp. 179-222). São Paulo: Martins Fontes.

Klinger, L.G., O'Kelley, S.E., Mussey, J.L., Goldstein, S., \& DeVries, M. (2012). Assessment of intellectual functioning in autism spectrum disorder. In D.P. Flanagan \& P.L. Harrison (Orgs.), Contemporary intellectual assessment (pp. 670-686). New York: The Guilford Press.

Lara, M.G. (2009). Atencion educativa a los alumnos/as com discapacidad visual. Revista digital Enfoques Educativos, 51, 136-146.

Lobato, M.J. (2005). Déficits Visuais. Recuperado de www. deficienciavisual.com/site/page4/files/defices_visuais.pdf.

Machado, E.V. (2011). A importância do (re) conhecimento do Sistema Braille para a humanização das políticas públicas de Inclusão. International Studies on Law and Education, 9, 49-54.

Malta, J., Endriss, D., Rached, S., Moura, T., \& Ventura, L. (2006). Desempenho funcional de crianças com deficiência visual, atendidas no Departamento de Estimulação Visual da Fundação Altino Ventura. Arquivo Brasileiro de Oftalmologia, 69(4), 571-574. doi: http://dx.doi.org/10.1590/S000427492006000400021

Masini, E. F. S. (1995). Algumas questões sobre a avaliação do portador de deficiência visual. Revista Brasileira de Estudos de Pedagogia, 76(184), 615-634.

McGrew, K. S., \& Flanagan, D. P. (1998). The intelligence test desk reference (ITDR): Gf-Gc Cross-Battery assessment. Boston: Allyn \& Bacon.

McGrew, K.S. (2009). CHC theory and the human cognitive abilities project: Standing on the shoulders of the giants of psychometric intelligence research. Intelligence, 37(1), 1-10. doi: 10.1016/j.intell.2008.08.004

Monteiro, M.M.B., \& Montilha, R.C.I. (2010). Intervenção fonoaudiológica e deficiência visual: percepções de profissionais de equipe interdisciplinar. Medicina (Ribeirão Preto), 43(1), 11-19.

Montilha, R. C. I., Gasparetto, M. E. R. F., \& Nobres, M. I. R. S. (2002). Deficiência Visual e Inclusão Escolar. In M. S Palhares, \& S. Marina, Escola Inclusiva (pp. 187-197). São Carlos, SP: Edufscar.

Nascimento, E., \& Flores Mendoza, C. E.. (2007). WISC-III e WAIS-III na avaliação da inteligência de cegos. Psicologia em Estudo, 12(3), 627-633. doi: http://dx.doi.org/10.1590/ S1413-73722007000300020
Nicolaiewsky, C.A., \& Correa, J. (2009). Habilidades cognitivolinguísticas e segmentação lexical em Braille. Revista Paidéia, 19(44), 341-348. doi: http://dx.doi.org/10.1590/S0103863X2009000300008

Nunes, S., \& Lomônaco, J.F.B. (2008). Desenvolvimento de conceitos em cegos congênitos: caminhos de aquisição do conhecimento: Desenvolvimento de conceitos em cegos congênitos. Psicologia Escolar e Educacional, 12(1), 119-138. doi: http://dx.doi.org/10.1590/S1413-85572008000100009.

Nunes, S., \& Lomônaco, J.F.B. (2010). O aluno cego: preconceitos e potencialidades. Psicologia Escolar e Educacional, 14(1), 55-64. doi: http://dx.doi.org/10.1590/S141385572010000100006

Pereira, M.L.D. (2009). Design inclusivo - um estudo de caso: Tocar para ver - brinquedos para crianças cegas e baixa visão. Dissertação de Mestrado, Universidade do Minho, SDUM, Portugal.

Primi, R., \& Almeida, L.S. (2000). Estudo de validação da bateria de provas de raciocínio (BPR-5). Psicologia: Teoria e Pesquisa, 16(2), 165-173. doi: http://dx.doi.org/10.1590/S010237722000000200009

Rabello, S., Motti, T.F.G., \& Gasparetto, M.E. R.F. (2007). Avaliação educacional por meio do teste IAR em escolares com cegueira. Revista Brasileira de Educação Especial, 13(2), 281-290. doi: http://dx.doi.org/10.1590/S1413-65382007000200009.

Roberts, A. (1996). Reabilitação Psicossocial do Cego. João Pessoa, PB: Editora Universitária.

Schelini, P. W., \& Wechsler, S.M. (2005). Bateria Multidimensional de Inteligência Infantil: desenvolvimento de instrumento. Psico-USF (Impresso), 10(2), 129-139. doi: http://dx.doi. org/10.1590/S1413-82712005000200004.

Schelini, P. W., \& Wechsler, S.M. (2006). Estudo da estrutura fatorial da bateria multidimensional de inteligência infantil. Estudos de Psicologia (Campinas), 23(2), 105-112. doi: http:// dx.doi.org/10.1590/S0103-166X2006000200001

Schneider, W.J., \& McGrew, K.S. (2012). The Cattell-Horn-Carroll model of intelligence. In D.P. Flanagan \& P.L. Harrison (Orgs.), Contemporary intellectual assessment (pp. 99-144). New York: The Guilford Press.

Wasserman, J.D. (2012). A history of intelligence assessment: the unfinished tapestry. In D.P. Flanagan, \& P.L. Harrison (Orgs.), Contemporary intellectual assessment (pp. 3-55). New York: The Guilford Press.

Wechsler, S.M., Nakano, T.C., Nunes, M.F.O., \& Minervino, C.A.S.M. (2010) Avaliação Cognitiva de crianças e jovens: aspectos multidimensionais. In C.S. Hutz. Avanços em Avaliação Psicológica e Neuropsicológica de crianças e adolescentes (pp. 31-68). São Paulo: Casa do Psicólogo. 